\title{
IMPROJECTIVE OPERATORS AND IDEALS IN \\ A CATEGORY OF BANACH SPACES
}

\author{
E. TARAFDAR
}

(Received 8 June 1970)

Communicated by G. E. Wall

\section{Introduction}

Kato [3] has introduced a class of operators called strictly singular operators. These operators have many properties in common with compact operators. In fact the concept of a strictly singular operator is an extension of the concept of a compact operator. Kato has proved that if $X$ and $X^{\prime}$ are Banach spaces, then the strictly singular operators of $X$ into $X^{\prime}$ form a closed subspace of the space of bounded linear operators of $X$ into $X^{\prime}$ and if $X=X^{\prime}$, then these operators form a two-sided ideal in the ring of bounded linear operators on $X$. He has also shown that the Riers-Schauder theorem holds for the spectrum of a strictly singular operator. Gohberg,Feldman and Markus [23] have treated the same class of operators with an equivalent definition.

Goldberg and Thorp have observed that the adjoint of a strictly singular operator is not necessarily strictly singular. Whitley [4] has dealt with the conjugates of such operators. Pelczynski [6] has introduced a dual concept to that of a strictly singular operator, called a strictly cosingular operator. He has proved that for Banach spaces $X$ and $X^{\prime}$, a bounded linear operator $T: X \rightarrow X^{\prime}$ is strictly singular (cosingular) if $T^{*}$ is strictly cosingular (singular) and $T^{*}$ is strictly singular (cosingular) if $T$ is strictly cosingular (singular) and $X$ is reflexive.

In section 1 of this paper we have introduced the concept of improjective operators. We have shown that the class of improjective operators is larger than the classes of strictly singular and strictly cosingular operators and that the improjective operators on a Banach space $X$ form a two-sided ideal in the multiplicative semigroup of bonded linear operators on $X$. We have also proved that for Banach spaces $X$ and $X^{\prime}$, a bounded linear operator $T: X \rightarrow X^{\prime}$ is improjective if $T^{*}$ is improjective and that $T^{*}$ is improjective if $T$ is improjective and $X$ reflexive. The other important results of this section are (i) an improjective 
operator $T: X \rightarrow X^{\prime}$ is strictly singular if $X^{\prime}$ is subprojective, and (ii) an improjective operator $T: X \rightarrow X^{\prime}$ is compact if $X$ and $X^{\prime}$ are Hilbert spaces.

In section 2 we consider some special Banach spaces $X$ and $X^{\prime}$ such that every bounded linear operator of $X$ into $X^{\prime}$, or of $X^{\prime}$ into $X$ is improjective. For example,

(a) if $X$ is a Banach space containing no copy of $c_{0}$ and $S$ a compact Hausdorff topological space, every bounded linear operator of $X$ into $C(S)$ is improjective;

(b) if $X$ is either a reflexive Banach space or a Banach space containing no copy of $l$, and $X^{\prime}$ is a Banach space isomorphic to an abstract $L$-space, then every bounded linear operator of $X$ into $X^{\prime}$ or, of $X^{\prime}$ into $X$ is improjective;

(c) if $X$ is a Banach space isomoprhic to an abstract $L$-space and $S$ a compact Hausdorff topological space, then the same as in (b) is true for $X$ and $C(S)$; and

(d) if $X$ is a separable Banach space, the same as in (b) ir true for $X$ and $m$.

Lastly we have shown that every bounded linear operator of $m$ into $X$ is strictly singular where $X$ is one of the space $c_{0}, l^{p}(1 \leqq p<\infty), c_{0}(S), l^{p}(S)$, $(1 \leqq p<\infty), S$ being countable and $L_{p}(\mu)(2 \leqq p<\infty) \mu$ being a separable measure.

In the final section we have introduced the concept of an ideal in a category and defined a non-identity ideal in a category of Banach spaces. We have established that the improjective operators form the largest non-identity ideal in the category of all Banach spaces; strictly singular operators form the largest nonidentity ideal in the category of all subprojective spaces; compact operators form the largest non-identity ideal in the category of all Hilbert spaces whereas the compact operators form the largest ideal in the category of all separable Hilbert spaces. Finally, we have proved that for Banach spaces $X$ and $X^{\prime}$, the improjective operators of $X$ into $X^{\prime}$ form a closed set of the space of all bounded linear operators of $X$ into $X^{\prime}$.

The author wishes to thank Professor B. Abrahamson for his advice and constant encouragement during the preparation of this paper.

\section{Improjective operators}

A subspace $Y$ of a Banach space $X$ is said to be complemented in $X$ if it is closed, and there is a closed subspace $Y^{\prime}$ of $X$ such that for each $x$ in $X$ there exist a $y$ in $Y$ and $y^{\prime}$ in $Y^{\prime}$ such that $x=y+y^{\prime}$ and if $y+y^{\prime}=0$, then $y=y^{\prime}=0$.

The following result is well known (e.g. [1], p. 211 or [2], p. 155-156):

A closed subspace $Y$ of a Banach space $X$ is complemented in $X$ if and only if there is a projection (a linear idempotent operator) of $X$ onto $Y$.

By a projection we shall always mean a continuous projection. 
Every Banach space $X$ is complemented in itself as we have always $X=X \oplus\{0\}(\oplus$ denotes direct sum), the identity map on $X$ being the projection.

Definition. A bounded linear operator $T: X \rightarrow X^{\prime}$ of a Banach space $X$ into a Banach space $X^{\prime}$ will be said to be an improjective operator if there is no infinite dimensional closed subspace $M$ (not necessarily proper) of $X$ such that the map $M \rightarrow T M$ (i.e. the restriction of $T$ to $M$ ) is an isomorphism and $T M$ is complemented in $X^{\prime} . T M$ denotes the image of $M$ under $T$.

Clearly an isomorphism $T: X \rightarrow X^{\prime}$ of a Banach space $X$ onto $X^{\prime}$, $\left(\operatorname{dim} X=\operatorname{dim} X^{\prime}=\infty\right)$, the identity operator on an infinite dimensional Banach space $X$ and a projection of $X$ onto an infinite dimensional closedsub space $Y$ are not improjective.

The following definition is due to Kato [3] (also see [4] and [5]).

Definition. A bounded linear operator $T: X \rightarrow X^{\prime}$ of a Banach space $X$ into a Banach space $X^{\prime}$ is said to be strictly singular if, for no infinite dimensional closed subspace $M$ of $X$, is the restriction of $T$ to $M$ an isomorphism (i.e., the restriction of $T$ to $M$ has a bounded inverse). Pelczynski [6] has introduced a dual concept called a strictly cosingular operator.

Definition. An operator $T: X \rightarrow X^{\prime}$ of a Banach space into a Banach space $X^{\prime}$ is said to be strictly cosingular provided that for no infinite dimensional Banach space $Z$ do there exist epimorphisms (onto maps) $T_{1}: X \rightarrow Z$ and $T_{2}: X^{\prime} \rightarrow Z$ such that the diagram

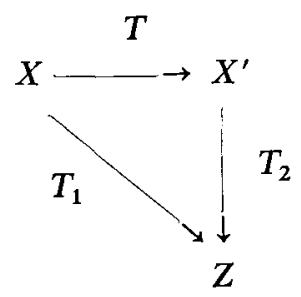

is commutative.

In the sequel, by a space $X$ or $X^{\prime}$ we shall always mean a Banach space, unless otherwise stated.

The proof of the following theorem is trivial.

THEOREM 1.1. Every strictly singular or strictly cosingular operator $T: X \rightarrow X^{\prime}$ is improjective. In fact we have the following relations:

$$
\text { compact } \searrow \text { strictly singular } \searrow \text { improjective. }
$$


There are operators which are improjective but not strictly singular or strictly cosingular.

ExAmple 1. Let $T$ be an isometric isomorphism of $l^{2}$ into $C[0,1]$. Then $T$ being an isomorphism is not strictly singular. To prove that $T$ is improjective we use the following result due to Grothendiek [7]:

No infinite dimensional closed reflexive subspace of $C[0,1]$ is complemented.

Suppose that $T$ is not improjective. Then there is an infinite dimensional closed subspace $M$ of $l^{2}$ such that the map $T: M \rightarrow T M$ is an isomorphism and $T M$ is complemented in $C[0,1]$. But $M$ being a closed subspace of $l^{2}$ is reflexive. Hence $T M$ is reflexive and thus by the above result cannot be complemented in $C[0,1]$. This proved that $T$ must be improjective. That the isometric isomorphism of $l^{2}$ into $C[0,1]$ (or $B(S), S$ an infinite set) is improjective also follows directly from our theorem 2.1 .

EXAMPLE 2. The injection map $T$ of $c_{0}$ into $m$ is not strictly singular. That $T$ is improjective follows from our theorem 2.5.

Example 3. Let $T$ be a bounded linear operator of $l$ onto $l^{2}$. Such a map $T$ always exists by a theorem of Banach and Mazur ([8], p. 211) and is strictly singular (see [5]). Hence $T$ is improjective by theorem 1.1. But $T$ being onto is not strictly cosingular.

In the following example we prove the existence of improjective operators which are neither strictly singular nor strictly cosingular.

EXAmple 4. Let $S$ be a set of the power of the continuum and $c_{0}(S), l(S)$, $m(S)$ be defined as in [9], p. 28. Then there exists an operator $T: l(S) \rightarrow c_{0}(S)$ of $l(S)$ onto $c_{0}(S)$ such that $T$ is strictly singular but $T^{*}: l(S) \rightarrow m(S)$ is neither strictly singular nor strictly cosingular (Pelczynski [6], ex. 1, p. 39-40). Obviously $T^{* *}: m(S)^{*} \rightarrow m(S)$ is also neither strictly singular nor strictly cosingular by virtue of prop. 3(a) of [6]. We shall prove that $T^{*}$ and $T^{* *}$ are improjective. We note that by a result of Phillips [10] (also see [9], p. 94) $m(S) \in \beta_{1}{ }^{(1)}$ and hence there is an isometirc $j$ of $m(S)$ onto a space $C\left(S_{1}\right)$ where $S_{1}$ is compact, Hausdorff and extremally disconnected ([9], p. 95). We also note that $m(S)^{*}$ is an abstract $L$-space ([9], p. 104). Thus by theorem 2.4 of section $2, j T^{* *}=T_{1}$, say, is improjective. Hence $T^{* *}=j^{-1} T_{1}$ is improjective by theorem 1.2 and by our theorem $1.5, T^{*}$ is improjective.

The above examples show that the class of improjective operators is larger than the class of strictly singular and strictly cosingular operators.

We now prove the following useful lemma.

(1) For $\lambda \geqq 1$, a Banach space $B$ is said to belong to $\beta_{\lambda}$ if for each space $B^{\prime} \supseteq B$, there is a projection $P$ of $B^{\prime}$ onto $B$ with $\|P\| \leqq \lambda$. 
LEMMA 1.1. If $T: X \rightarrow X^{\prime}$ is a bounded linear operator such that the restriction of $T$ to a closed subspace $M$ of $X$ is an isomorphism, and TM is complemented in $X^{\prime}$, then $M$ is complemented in $X$.

Proof. Let $T^{\prime}$ denote the 'restriction of $T$ to: $M$, and $T^{\prime-1}: T M \rightarrow M$ denote the operator inverse to $T^{\prime}$. Now since $T M$ is complemented in $X^{\prime}$, there is a projection $P$ of $X^{\prime}$ onto $T M$. We define the bounded linear operator $P^{\prime}: X \rightarrow M$ by $P^{\prime}=T^{\prime-1} P T$. Now $P^{\prime 2}=T^{\prime-1} P T T^{\prime-1} P T=T^{\prime-1} P^{2} T=T^{-1} P T=P^{\prime}$. Thus $P^{\prime}$ is a projection of $X$ onto $M$. Hence $M$ is complemented in $X$.

THEOREM 1.2. If $T: X \rightarrow X^{\prime}$ is improjective then $T T_{1}$ and $T_{2} T$ are also improjective wehre $T_{1}: X_{1} \rightarrow X$ and $T_{2}: X^{\prime} \rightarrow X_{2}$ are arbitrary bounded linear operators.

Proof. If possible, let $T T_{1}$ be not improjective. Then there is an infinite dimensional closed subspace $M$ of $X_{1}$ such that the restriction of $T T_{1}$ to $M$ is an isomorphism and $T T_{1} M$ is complemented in $X^{\prime}$. Since the restriction of $T T_{1}$ to $M$ is an isomorphism, $\left\|T T_{1} x\right\| \geqq \gamma\|x\|, \gamma>0$, for all $x \in M$. Set $T_{1} M=M^{\prime}$. Now $\left\|T_{1} x\right\| \geqq \gamma\|T\|^{-1}\|x\|$, for all $x \in M$. Thus $M^{\prime}=T_{1} M$ is infinite dimensional and closed. Now for any $y \in M^{\prime}$, there is a unique $x \in M$ such that $T_{1}(x)=y$. Hence

$$
\|T y\|=\left\|T T_{1} x\right\| \geqq \gamma\|x\| \geqq \gamma\left\|T_{1}\right\|^{-1}\|y\| \text { as }\|y\|=\left\|T_{1} x\right\| \leqq\left\|T_{1}\right\|\|x\| .
$$

This is true for all $y \in M^{\prime}$. Thus the restriction of $T$ to $M^{\prime}$ is an isomorphism and $T M^{\prime}=T T_{1} M$ is compemented in $X^{\prime}$ by hypothesis. These together contradict that $T$ is improjective.

Next, if possible, let $T_{2} T$ be not improjective. Then there is an infinite dimensional closed subspace $M_{1}$ of $X$ such that $\left\|T_{2} T x\right\| \geqq \gamma_{1}\|x\|, \gamma_{1}>0$, for all $x \in M_{1}$ and $T_{2} T M_{1}$ is complemented in $X_{2}$. As before we have

and

$$
\|T x\| \geqq \gamma_{1}\left\|T_{2}\right\|^{-1}\|x\|, \text { for all } x \in M_{1}
$$

$$
\left\|T_{2} y\right\| \geqq \gamma_{1}\|T\|^{-1}\|y\|, \text { for all } y \in T M_{1} .
$$

(1) implies that the restriction of $T$ to $M_{1}$ is an isomorphism and $T M_{1}=M_{1}^{\prime}$ is closed and infinite dimensional. (2) implies that the restriction of $T_{2}$ to $M_{1}^{\prime}$ is an isomorphism. Again since $T_{2} M_{1}^{\prime}$ is complemented in $X_{2}, M_{1}^{\prime}$ is by lemma 1.1 complemented in $X^{\prime}$. Thus we have that the restriction of $T$ to $M_{1}$ is an isomorphism and $T M_{1}=M_{1}^{\prime}$ is complemented in $X^{\prime}$. But these contradict that $T$ is improjective.

The following definition of subprojective spaces is due to Whitley [4].

Definition. A Banach space $X$ is subprojective if, for every infinite dimen- 
sional closed subspace $M$ of $X$, there exists an infinite dimensional closed subspace $N$ contained in $M$ and a projection of $X$ onto $N$.

Example of subprojective spaces: The Hilbert spaces and the spaces $l^{p}$ $(1 \leqq p<\infty), c_{0}, L_{p}(2 \leqq p<\infty), l^{p}(S)(1 \leqq p<\infty)$ and $c_{0}(S)$ are subprojective. (For proof see [4] and [1] and for definition of $l^{p}(S)$ and $c_{0}(S)$ see [9], p. 28).

THEOREM 1.3. If $T: X \rightarrow X^{\prime}$ is improjective and $X^{\prime}$ is sub-projective, then $T$ is strictly singular.

Proof. If possible, let $T$ be not strictly singular. Then we can find an infinite dimensional closed subspace $M$ of $X$ such that the restriction of $T$ to $M$ is an isomorphism. Since $T M$ is closed and $X^{\prime}$ is subprojective, there is an infinite dimensional closed subspace $N$ of $T M$ such that there is a projection of $X^{\prime}$ onto $N$, i.e. $N$ is complemented in $X^{\prime}$. Set $T^{-1}(N) \cap M=M^{\prime}$. Then $M^{\prime}$ is an infinite dimensional closed subspace of $X$ such that the restriction of $T$ to $M^{\prime}$ has a bounded inverse and $T M^{\prime}=N$ is complemented in $X^{\prime}$. These contradict that $T$ is improjective. Hence $T$ is strictly singular.

Corollary 1.1. If $T: X \rightarrow X^{\prime}$ is strictly cosingular and $X^{\prime}$ is subprojective, then $T$ is strictly singular.

ProOF. $T$ being strictly cosingular is improjective. Hence the corollary follows from the theorem 1.3.

CoRollary 1.2. Every improjective operator $T: X \rightarrow X^{\prime}$ of a Hilbert space $X$ into a Hilbert space $X^{\prime}$ is compact.

Proof. As $X^{\prime}$ is subprojective, $T$ is strictly singular by theorem 1.3 and by a result of Kato ([3], see remark p. 287-288) $T$ is compact.

Definition. A Banach space $X$ is superprojective if, for every closed subspace $M$ with infinite co-dimension (i.e. $\operatorname{dim} X / M$ ), there exists a closed subspace $N$ of infinite co-dimension containing $M$ and a projection of $X$ onto $N$.

The spaces $l^{p}(S)(1<p<\infty)$ and $L_{p}(1<p \leqq 2)$ are superprojective. (See [4]).

THEOREM 1.4. If $X$ is reflexive and $Y$ is superprojective, then every improjective operator $T: X \rightarrow Y^{*}$ is strictly singular.

Proof. Suppose that $T$ is not strictly singular. Then we can find an infinite dimensional closed subspace $M$ of $X$ such that the restriction of $T$ to $M$ has a bounded inverse. Since $X$ is reflexive, $M$ is reflexive. Hence $T M$ is an infinite dimensional closed reflexive subspace of $Y^{*}$. Now by theorem 4.6 of [4] there 
is an infinite dimensional closed subspace $N$ contained in $T M$ such that $N$ is complemented in $Y^{*}$. Setting

$$
T^{-1}(N) \cap M=M^{\prime}
$$

and considering the restriction of $T$ to $M^{\prime}$ we conclude that $T$ is not improjective, which is a contradiction. This provers the theorem.

COROLlaRY 1.3. If $X$ is a Banach space and $Y$ a reflexive superprojective space, then every improjective operator $T: X \rightarrow Y^{*}$ is strictly singular.

Proof. By corollary 4.7 of $[4], Y^{*}$ is sub-projective. Hence the corollary follows from the theorem 1.3 .

THEOREM 1.5. If $T: X \rightarrow X^{\prime}$ is a bounded linear operator such that $T^{*}: X^{*} \rightarrow X^{*}$ is improjective then $T$ is improjective.

Proof. If possible, let $T$ be not improjective. Then there is an infinite dimensional closed subspace $M$ of $X$ such that the restricted map $M \rightarrow T M$ is an isomorphism and $T M$ is complemented in $X^{\prime}$. By lemma $1.1 M$ is also complemented in $X$ and there is a projection $P^{\prime}$ of $X$ onto $M$ where $P^{\prime}$ is given by $P^{\prime}=T^{\prime-1} P T$ where $P$ is the projection of $X^{\prime}$ onto $T M$ and $T^{\prime}$ is the restriction of $T$ to $M$ (see lemma 1.1$)$.

Thus we can write

and

$$
X=M \oplus M^{\prime} \text { where } M^{\prime} \text { is a closed subspace of } X
$$

$$
X^{\prime}=T M \oplus N \text { where } N \text { is a clored subspace of } X^{\prime} \text {. }
$$

We have also ([2]. p. 156)

and

$$
X^{*}=M^{\perp} \oplus M^{\perp}
$$

$$
X^{\prime *}=(T M)^{\perp} \oplus N^{\perp}
$$

Now since $P$ is an onto map, $P^{*}$ is a one-to-one bounded linear operator onto an infinite dimensional closed subspace $P^{*}(T M)^{*}=Z$ of $X^{\prime *}$ (see e.g. [11], p. 237). Since the restriction of $T$ to $M$ has a bounded inverse, the restriction of $T^{*}$ to $Z$ has a bounded inverse (see the proof of the theorem 2.2 in [4]). We prove that $T^{*} Z$ is complemented in $X^{*}$. Since $P$ has a closed range, $Z=N^{\perp}$ $N$ being the null space of $P$. Similarly considering the projection $P^{\prime}$ of $X$ we can show that $P^{\prime *} M^{*}=M^{\prime \perp}$. We have

$$
P^{\prime}=T^{\prime-1} P T
$$

Hence $P^{\prime *}=T^{*} P^{*}\left(T^{\prime-1}\right)^{*}$ i.e., 


$$
P^{*} M^{*}=T^{*} P^{*}\left(T^{\prime-1}\right)^{*} M^{*}=T^{*} P^{*}(T M)^{*}
$$

as $\left(T^{\prime-1}\right)^{*}$ maps $M^{*}$ onto $(T M)^{*}$.

Hence $M^{\perp}=T^{*} Z$. Thus $T^{*} Z$ is complemented in $X^{*}$. Thus the restriction of $T^{*}$ to $Z$ has a bounded inverse and $T^{*} Z$ is complemented in $X^{*}$. These together contradict that $T^{*}$ is improjective.

Corollary 1.4. If $T: X \rightarrow X^{\prime}$ is improjective and $X$ is reflexive, then $T^{*}$ is improjective.

Proof. Let $J$ and $J^{\prime}$ be respectively the canonical embeddings of $X$ onto $X^{* *}$ and $X^{\prime}$ into $X^{* * *}$. Then $T^{* *}=J^{\prime} T J^{-1}$. Hence $T^{* *}$ is improjective by theorem 1.2 as $T$ is improjective. Hence by the theorem $1.5 T^{*}$ is improjective.

That the above corollary is not always true if the reflexivity on $X$ is removed can be seen from the following example.

EXAMPLE 5. $B_{n}$ is the space of $R$. C. James ([12], theorem 2) with the property that the $2 n$th conjugate space $B_{n}^{(2 n)}$ of $B_{n}$ is separable and contains a complemented subspace $E$ isomorphic to $l$. Since $B_{n}^{(2 n)}$ is separable, $B_{n}$ is separable. Hence there is a bounded linear operator $T$ mapping $l$ onto $B_{n}([8]$, p. 211). Pelczynski ([6], Ex. 3, p. 40) has proved that

(i) $T^{(2 n-1)}$, the $(2 n-1)$ th adjoint operator to $T$ is strictly co-singular;

(ii) $T^{(2 n)}$ is neither strictly singular nor strictly cosingular; and $j P T^{(2 n)}: L(v)$ $\rightarrow l$ is not strictly singular, $\left({ }^{2}\right)$ where $P$ is the projection of $B_{n}^{(2 n)}$ onto $E, j$ an isomorphism between $E$ and $l$ and $L(v)$ conjugate space of even order to $l$.

Now, $T^{(2 n-1)}$ being strictly cosingular, is improjective. If possible, let $T^{(2 n)}$ be improjective. Then $j P T^{(2 n)}$ is improjective. Then, since $l$ is subprojective $j P T^{(2 n)}$ is by theorem 1.3 strictly singular which contradicts the above result of Pelczynski. Hence $T^{(2 n)}$ is not improjective.

\section{Improjestive operators on some special Banach spaces}

We consider the following spaces (for definitions see [13]).

(1) $C(S), S$ a compact Hausdorff space;

(2) $B(S), S$ a set;

(3) $L_{\infty}(S, \Sigma, \mu)$ and $L(S, \Sigma, \mu),(S, \Sigma, \mu)$ a positive measure;

(4) $r c a(S)$ and $b a(S)$, which are isometrically isomorphic to the conjugate spaces of, respectively, $C(S)$ and $B(S)$;

(5) Any complimented subspace of the spaces (1)-(4).

(2) $v$ is a non-trivial measure defined on a field $\Sigma$ of all Borel subsets of a topological space $X$ and $L(v)=L(X, \Sigma, v)$ is the Banach space of all $v$-equivalency classes of $v$-measurable and $v$-absolutely summable scalar valued functions on $X$ (see [13], Chap. IV). 
Whitley [4] has proved that every bounded linear operator of a space $X$ of (1)-(5) into a reflexive space $Y$ is strictly singular.

LEMMA 2.1. If $X$ is any of the spaces (1)-(5) above, and $Y$ an infinite dimensional, reflexive closed subspace of $X$, then $Y$ is not complemented in $X$.

Proof. Suppose that $Y$ is complemented in $X$. Then there is a projection $P$ of $X$ onto $Y$. Now $Y$ being reflexive, by Whitley's result mentioned above, $P$ is strictly singular. This implies that $Y$ is finite dimensional which is a contradiction.

THEOREM 2.1. Let $X$ be an arbitrary reflexive Banach space and $X^{\prime}$ any of the spaces (1)-(5) above. Then every bounded linear operator $T$ of $X$ into $X^{\prime}$ is improjective.

Proof. If possible, let $T$ be not improjective. Then there there must be an infinite dimensional closed subspace $M$ of $X$ such that the map $M \rightarrow T M$ is an isomorphism and $T M$ is complemented in $X^{\prime} . M$ being a closed subspace of a reflexive space is reflexive. Hence $T M$ is an infinite dimensional closed reflexive subspace of $X^{\prime}$. Hence by the above lemma 2.1,TM cannot be complemented in $X^{\prime}$. This is a contradiction.

THEOREM 2.2. Let $X$ be a Banach space containing no subspace isomorphic to $c_{0}$ and $S$ a compact Hausdorff topological space. Then every bounded linear operator $T$ of $X$ into $C(S)$ is improjective [and every bounded linear operator of $C(S)$ into $X$ is strictly singular (Pelczynski)].

Proof. If possible, let $T: X \rightarrow C(S)$ be not improjective. Then there is an infinite dimensional closed subspace $M$ of $X$ such that the map $M \rightarrow T M$ is an isomorphism and $T M$ is complemented in $C(S)$. Since $T M$ is complemented in $(C S)$ and $T M$ is infinite dimensional, by corollary $2^{(3)}$ of [1] $T M$ contains a closed subspace $N$ isomorphic to $c_{0}$. Set $T^{-1}(N) \cap M=M^{\prime}$. Now since the map $M^{\prime} \rightarrow T M^{\prime}=N$ is also an isomorphism, $M^{\prime}$ is isomorphic to $c_{0}$ which is a contradiction.

Pelczynski [6] has proved that a bounded linear operator $T$ of $C(S)$ into any Banach space $X$ is weakly compact if and only if $T$ is strictly singular. If $X$ is as above, then every bounded linear operator of $C(S)$ into $X$ is weakly compact ([1], theorem 5).

We note that if $X$ is as in theorem 2.2 and $S$ is compact, Hausdorff and dispersed (i.e. $S$ does not have any non-empty perfect set) then every bounded linear operator $T$ of $X$ into $C(S)$ is strictly singular. This is because every infinite dispensional closed subspace of $C(S)$ contains a closed subspace isomorphic to $c_{0}([14]$, p. 214).

(3) Corollary 2 of [1] states that if $S$ is a compact Hausdorff topological space and $X$ a closed subspace complemented in $C(S)$, then either $X$ contains a subspace isomorphic to $c_{0}$ or $X$ is of finite dimension. 
THeOREM 2.3. Let $X$ be a Banach space which is either reflexive or does not contain any closed subspace isomorphic to $l$ and $X^{\prime}$ a Banach space isomorphic to any abstract $L$-space. Then every bounded linear operator $T$ of $X$ into $X^{\prime}$, or of $X^{\prime}$ into $X$ is improjective.

Proof. If $T$ maps $X$ into $X^{\prime}$ and is not improjective, then there is an infinite dimensional closed subspace $M$ of $X$ such that the map $M \rightarrow T M$ is an isomorphism and $T M$ is complemented in $X^{\prime}$. Now by corollary $4^{(4)}$ of [1],TM contains a closed subspace $N$ which is isomorphic to $l$. The closed subspace

$$
T^{-1}(N) \cap M=M^{\prime}
$$

of $X$ is then obviously isomorphic to $l$ which is impossible.

Next let $T$ map $X^{\prime}$ into $X$ and $T$ be not improjective. Then there is an infinite dimensional closed subspace $M_{1}$ of $X^{\prime}$ such that the map $M_{1} \rightarrow T M_{1}$ is an isomorphism and $T M_{1}$ is complemented in $X$. By lemma $1.1 M_{1}$ is complemented in $X^{\prime}$. Hence again by corollary 4 of [1], $M_{1}$ contains a closed subspace $N_{1}$ isomorphic to $l$. Thus $T N_{1}$ is isomorphic to $l$ which is impossible.

LEMMA 2.2. If $S$ is compact, metrizable and dispersed, then $C(S)$ is subprojective.

Proof. By the result of [14], p. 214 it follows that every infinite dimensional closed subspace $M$ of $C(S)$ contains a closed subspace $N$ isomorphic to $c_{0}$. Again since $S$ is compact and metrizable, $C(S)$ is separable. Hence by a result of Sobczyk [15] (also see [1], theorem 4) $N$ is complemented in $C(S)$. Thus $C(S)$ is subprojective.

CoRollary 2.1. If $X$ is a Banach space isomorphic to an abstract L-space and $S$ is compact, metrizable and dispersed, then every bounded linear operator of $X$ into $C(S)$ is strictly singular and every bounded linear operator of $C(S)$ into $X$ is improjective.

Proof. By the result of [14], p. 214, no subspace of $C(S)$ is isomorphic to $l$. Hence by theorem 2.3 every bounded linear operator $T$ of $X$ into $C(S)$ or, of $C(S)$ into $X$ is improjective. Now, since $C(S)$ is subprojective by the above lemma 2.2, $T$ is strictly singular by theorem 1.3 when $T$ maps $X$ into $C(S)$.

The above corollary remains valid if we replace $C(S)$ by $c_{0}$. For we have $c_{0} \simeq c=C(S)$ where $S$ is the one point compactification of the integers. Now

(4) Corollary 4 of [1] states that if $X$ is a Banach space isomorphic to an abstract $L$-space and $M$ is a closed subspace complemented in $X$, then either $M$ contains a complemented subspace which is isomorphic of $l$ or $M$ is to finite dimension. 
let $T$ be any bounded linear operator of $X$ into $c_{0}$ or of $c_{0}$ into $X$ and $j$ the isomorphism of $c_{0}$ onto $C(S)$. Then $j T=A$ or $T j^{-1}=A^{\prime}$ is by the above corollary strictly singular or improjective. Hence $T=j^{-1} A$ or, $T=A^{\prime} j$ is respectively strictly singular or improjective.

THEOREM 2.4. If $X$ is a Banach space isomorphic to an abstract L-space and $S$ a compact Hausdoeff topological space, then every bounded linear operator $T$ of $X$ into $C(S)$, or of $C(S)$ into $X$ is improjective.

Proof. If $T$ maps $X$ into $C(S)$ and $T$ is not improjective, then there is an infinite dimensional closed subspace $M$ of $X$ such that the map $M \rightarrow T M$ is an isomorphism and $T M$ is complemented in $C(S)$. Hence by lemma $1.1, M$ is also complemented in $X$. Thus we have

$$
X=M \oplus M^{\prime} \text { for some closed subspace } M^{\prime} \text { of } X
$$

and $C(S)=T M \oplus N$ for some closed subspace $N$ of $C(S)$. Now by corollary 4 of [1] (already mentioned in theorem 2.3) $M$ contains a complemented closed subspace $M_{1}$ isomorphic to $l$. Since $M_{1}$ is complemented in $X, M_{1}$ is also complemented in $M$ (if $P$ is the projection of $X$ onyo $M_{1}$, then the restriction of $P$ to $M$ is the required projection of $M$ onto $M_{1}$ ). Thus $M=M_{1} \oplus M_{2}$ for some closed subspace $M_{2}$ contained in $M$. Now since the restriction of $T$ to $M$ has a bounded inverse, it is easy to check that $T M=T M_{1} \oplus T M_{2}$. Thus we can write $C(S)=\left(T M_{1} \oplus T M_{2}\right) \oplus N$. Hence $T M_{1}$ is complemented in $C(S)$ (composition of two projections is a projection). Now by corollary 2 of [1] (mentioned in theorem 2.2) $T M_{1}$ contains a closed subspace isomorphic to $c_{0}$. But $T M_{1}$ being isomorphic to $M_{1}$ is isomorphic to $l$. These together imply that $l$ contains a subspace isomorphic to $c_{0}$ which is impossible (see [16], chap. 12).

Next, let $T$ map $C(S)$ into $X$ and if possible, let $T$ be not improjective. Then there is an infinite dimensional closed subspace $Y$ of $C(S)$ such that the map $Y \rightarrow T Y$ is an isomorphism and $T Y$ is complemented in $X$. By corollary 4 of [1] $T Y$ contains a complemented subspace $Z$ isomorphic to $l$. Set $T^{-1}(Z) \cap Y=Z^{\prime}$. Then by lemma $1.1, Z^{\prime}$ is complemented in $C(S)$ as the map $Z^{\prime} \rightarrow T Z^{\prime}=Z$ is also an isomorphism. Now by corollary 2 of [1], $Z^{\prime}$ contains a closed subspace isomorphic to $c_{0}$. But $Z^{\prime}$, being isomorphic to $Z$, is isomorphic to $l$. These will lead to a contradiction as above.

THEOREM 2.5. If $X$ is a separable Banach space, then every bounded linear operator $T$ of $X$ into $m$, or of $m$ into $X$ is improjective where $m$ is the space of all bounded sequences.

Proof. Let $T$ map $X$ into $m$. If $T$ is not improjective, then there is an infinite dimensional closed subspace $M$ of $X$ such that the map $M \rightarrow T M$ is an isomorphism and $T M$ is complemented in $m$. Then by corollary 5 of [1] which states that no infinite dimensional complemented subspace of $m$ is separable, 
$T M$ is not separable. This implies that $M$ is not separable which is impossible. Thus $T$ is improjective.

If $T$ maps $m$ into $X$, then by applying the same corollary 5 of [1] and our lemma 1.1 we can prove that $T$ is improjective.

Corollary 2.2. Every bounded linear operator $T$ of $m$ into a separable subprojective space $X$ is strictly singular.

Proof. $T$ is, by the above theorem 2.5 , improjective. Hence by theorem 1.3 $T$ is strictly singular as $X$ is subprojective.

Thus every bounded linear operator of $m$ into $c_{0}, l^{p}(1 \leqq p<\infty)$, $L_{p}(S, \Sigma, \mu)(2 \leqq p<\infty)$ where $\mu$ is separable measure (see [17], p. 75), $l^{p}(S)$ $(1 \leqq p<\infty)$ or $c_{0}(S), S$ being countable in the latter two cases (see [9], p. 29) is strictly singular.

Now by using the theorem 2 of [1] (which states that every infinite dimensional complemented subspace of $c_{0}$ is isomorphic to $c_{0}$ ) and our theorem 1.3 we can prove the following theorem.

THEOREM 2.6. Let $X$ be as in theorem 2.2. Then every bounded linear operator of $X$ into $c_{0}$ is strictly singular and every bounded linear operator of $c_{0}$ into $X$ is improjective.

We can also deduce the above theorem as a corollary of our previous theorem 2.2.

REMARK. The above result as to strictly singular operators is already known [18] and ours is only a new proof. In fact, Lacey and Whitley have the stronger result ([18], p. 3):

Every bounded linear operator of $c_{0}$ into $X$ is compact iff $X$ contains no copy of $c_{0}$, iff every bounded linear operator of $c_{0}$ into $X$ (or of $X$ into $c_{0}$ ) is strictly singular.

However, the converse of the statement in $(\cdot)$ is not true, i.e., if every bounded linear operator of $X$ into $c_{0}$ is strictly singular, then it does not imply that $X$ contains no copy of $c_{0}$. In fact, we have proved (corollary 2.2) that every bounded linear operator of $m$ into $c_{0}$ is strictly singular. Clearly $m$ contains $c_{0}$.

In a letter, H. E. Lacey has pointed out that the converse of the above would be true if $X$ is taken to be separable (in this case the above result can even be slightly generalized by taking $C(S)$ in place of $c_{0}$ where $S$ is compact, metrizable and dispersed (infinite)). The proof is as follows.

Combining the results of Pelczynski ([6], theorem 1) and ([1] theorem 5), and Lacey and Morris ([19] theorem 5), it can be proved that if $S$ is compact, 
infinite, metrizable and dsipersed and $X$ any Banach space, then the following four statements are equivalent:

1) $X$ contains no copy of $c_{0}$;

2) Every bounded linear operator $T: C(S) \rightarrow X$ is weakly compact;

3) Every bounded linear operator $T: C(S) \rightarrow X$ is compact;

4) Every bounded linear operator $T: C(S) \rightarrow X$ is strictly singular.

Since $c_{0} \simeq c=C(S)$ where $S$ is the one point compactification of the integers, the above four statements will remain equivalent if we replace $C(S)$ by $c_{0}$.

If $X$ contains no copy of $c_{0}$, then every bounded linear operator $T: X \rightarrow c_{0}$ is strictly singular by our theorem 2.5 and every bounder linear operator $T: X \rightarrow C(S)$ is strictly singular by the note following theorem 2.2 .

Now if $X$ is separable and every bounded linear operator $T: X \rightarrow C(S)$ or $c_{0}$ is strictly singular, then $X$ contains no copy of $c_{0}$. For, if $X$ contains a copy of $c_{0}$, then by the result of Sobczyk, $c_{0}$ is complemented in $X$, i.e. there is a projection $P$ of $X$ onto $c_{0}$. But $P$ and $j P: X \rightarrow C(S)$ where $j$ is the injection of $c_{0}$ into $C(S)$ are not strictly singular. Thus if $X$ is separable and $S$ is as above, then every bounded linear operator of $C(S)$ (or $c_{0}$ ) into $X$ is compact iff $X$ contains no copy of $c_{0}$, iff every bounded linear operator of $V(S)$ (or $c_{0}$ ) into $X$ (or of $X$ into $C(S)$ (or $\left(c_{0}\right)$ ) is strictly singular.

\section{Ideals in a category}

For the definition of a category, see [20]. The concept of ideals in a category introduced in this section differs from the known ones, e.g. Isbell [21], Kelly [22] and Sulgeifer [23] and [24].

Definition. In a category $\mathscr{C}$, a collection $I$ of morphisms will be said to form a $t$ wo-sided ideal or simply an ideal in $\mathscr{C}$ if $c d \in I$ and $d c^{\prime} \in I$, whenever $d \in I, c, c^{\prime} \in \mathscr{C}$ and $d c$ and $d c^{\prime}$ are defined in $\mathscr{C}$. An ideal $I$ in $\mathscr{C}$ is said to be proper if $I \neq \mathscr{C}$, and $I \neq \phi$.

EXAMPLE OF IDEALS. In a category of Banach spaces and bounded linear operators, the completely continuous operators, the finite-dimensional operators, the strictly singular operators, the improjective operators introduced in the section 1, form ideals. In a category of topological spaces and continuous maps, the collection of maps $f: X \rightarrow Y$ such that $f(X)$ is relatively compact in $Y$ : in a category of sets and maps the collection of maps having finite images; in a category of groups and homomorphisms, the collection of homomorphisms having abelian images; in a category of pairs $A \subset B$ of sets and morphisms $f: A \subset B \rightarrow A_{1} \subset B_{1}, f(A) \subset A_{1}, f(B) \subset B_{1}$ the collection of morphisms $f$ such that $f(B) \subset A_{1}$, are further examples of ideals.

Zero maps in every category with zero objects trivially form an ideal. Any 
ideal different from the zero ideal is called a non-zero ideal. In what follows, by 'ideal' we shall mean 'non-zero ideal'.

A proper ideal in a category $\mathscr{C}$ is said to be largest if it contains every proper ideal of $\mathscr{C}$ and smallest if it is contained in every ideal of $\mathscr{C}$.

Our aim in this section is to study some of the ideals in a category of Banach or Hilbert spaces. Our morphisms in either case will always be bounded linear operators (the domain of an operator being the entire space in which the operator is defined).

DEFINITION. A category $\mathscr{B}$ of Banach spaces is said to be regular if for every $X \in \mathscr{B}, X^{*} \in \mathscr{B}$.

The proof of the following theorem is easy and can be omitted.

THEOREM 3.1. If $I$ is a proper ideal in a regular category $\mathscr{B}$ of reflexive Banach spaces, then $I^{*}$ is also a proper ideal in $\mathscr{B}$ where

$$
I^{*}=\left\{T \in \mathscr{B}: T^{*} \in I\right\}
$$

and $T^{*}$ is the conjugate (adjoint) operator of $T$.

THEOREM 3.2. For an arbitrary ideal $I$ in a category $\mathscr{H}$ of Hilbert spaces, $I=I^{*}$.

Proof. Let $T: X \rightarrow X^{\prime}$ be an arbitrary bounded linear operator belonging to $I$. We consider $|T|=\left(T^{*} T\right)^{\frac{1}{2}}$ which is a self-adjoint operator on $X$. By the polar decomposition theorem (e.g. see [25], p. 4), there is a partially isometric operator $U: X \rightarrow X^{\prime}$ such that $T=U|T|$ and $|T|=U^{*} T$. Hence $|T| \in I$ as $T \in I$. Now $T^{*}=|T| U^{*} \in I$ as $|T| \in I$. Hence $T \in I^{*}$. Thus $I \subset I^{*}$. Similarly we can prove that $I^{*} \subset I$. Hence $I=I^{*}$.

Calkin [26] has a similar result for an ideal in a ring of operators on a Hilbert space.

COROLLARY 3.1. If a bounded linear operator $T$ mapping a Hilbert space $X$ into a Hilbert space $X^{\prime}$ has a range of finite dimension $n$ or an infinite dimension of cardinality $\alpha\left(\geqq \chi_{0}\right)$, then $T^{*}$ has also a range of the same dimension, i.e. $\operatorname{dim} R(T)=\operatorname{dim} R\left(T^{*}\right)$ where $R(T)$ is the range of $T$.

PRoof. Let $\mathscr{H}$ be the category of all Hilbert spaces. Then $T \in \mathscr{H}$. Suppose Suppose that $R(T)$ has a dimension $n$. Now all the bounded linear operators of dimension $\leqq n$ form an ideal $I$ in $\mathscr{H}$. Since $T \in I, T^{*} \in I$ by virtue of theorem 3.2 and hence $\operatorname{dim} R\left(T^{*}\right) \leqq n=\operatorname{dim} R(T)$. Similarly we can prove that $\operatorname{dim} R(T) \leqq \operatorname{dim} R\left(T^{*}\right)$. The rest of corollary 3.1 can be proved by repeating the above argument and noting that the collection of all bounded linear operators having ranges not containing closed subspaces of dimension of cardinality exceeding $\alpha$ forms an ideal in $\mathscr{H}$. 
The above corollary is not in general true if $X$ and $X^{\prime}$ are not Hilbert spaces. For example, we consider the bounded linear operator $T: l \rightarrow c_{0}$ mapping $l$ onto $c_{0}$ (such a map always exists by a theorem of Banach and Mazur ([8], p. 211) already mentioned in example 3). Now $T^{*}$ is an isomorphism of $l$ into $m$ and $T^{* *}$ maps $m^{*}$ onto $m$. Hence the cardinality of the dimension $R\left(T^{*}\right)$ is not equal to that of $R\left(T^{* *}\right)$.

Definition. An ideal $I$ in a category $\mathscr{B}$ of Banach spaces will be said to be a non-identity ideal if for every infinite dimensional Banach space $X \in \mathscr{B}$, $I_{X}$ (identity on $\left.X\right) \notin I$. The largest non-identity ideal is one which contains all other non-identity ideals.

From the above definition of non-identity ideal, it is obvious that a nonidentity ideal in a category having at least one infinite dimensional Banach space is always proper.

Throughout the rest of this paper we shall assume that a category $\mathscr{B}$ always contains at least one infinite dimensional space.

THEOREM 3.3. In a category $\mathscr{B}$ of Banach spaces the largest non-identity ideal $\mu$ always exist.

Proof. Let $\left\{I_{\alpha}\right\}$ be the family of all non-identity ideals in $\mathscr{B}$. This is not empty since the zero ideal belongs to it. Then $\mu=\bigcup_{\alpha} I_{\alpha}$ is obviously an ideal in $\mathscr{B}$. Let $X$ be an arbitrary infinite dimensional Banach space in $\mathscr{B}$. Then $I_{X} \notin \mu$. For if $I_{X} \in \mu$, then there will be at least one $\alpha$ such that $I_{X} \in I_{\alpha}$ which will contradict that $I_{\alpha}$ is a,non-identity ideal in $\mathscr{B}$. Thus by the definition of the largest ideal, $\mu$ is the largest non-dentity ideal in $\mathscr{B}$.

The proof of the following lemma is trivial.

LEMMA 3.1. If $I$ is a non-identity ideal in a regular category of reflexive Banach spaces, then $I^{*}$ is also a non-identity ideal in $\mathscr{B}$.

COROLlary 3.2. In a regular category $\mathscr{B}$ of reflexive Banach spaces, if $\mu$ is the largest non-identity ideal, then $\mu=\mu^{*}$.

Proof. From the above lemma 3.1, $\mu^{*} \subset \mu$. Next, let $T \in \mu$. Then $T^{*} \in \mu^{*} \subset \mu$. Hence $T=T^{* *} \in \mu^{*}$ as $T^{*} \in \mu$. Thus $\mu \subset \mu^{*}$.

Definition. A category $\mathscr{B}$ of Banach spaces is said to be hereditary if for every Banach space $X \in \mathscr{B}, Y \in \mathscr{B}$, whenever $Y$ is a closed subspace of $X$. The categories of all Banach spaces, all reflexive Banach spaces, all Hilbert spaces, all separable Hilbert spaces, all subprojective spaces (see [4]) are all hereditary.

LEMMA 3.2. If $I$ is an ideal in a category $\mathscr{B}$ of Banach spaces and $T: X \rightarrow X^{\prime}$ is in $I$, then the restriction $\hat{T}$ of $T$ to a closed subspace $Y$ of $X$ also $\in I$ whenever $Y \in \mathscr{B}$. 
Proof. Let $i$ be the injection map of $Y$ into $X$. Then obviously $\hat{T}=T i \in I$ as $T \in I$ and $i \in \mathscr{B}$.

THEOREM 3.4. In a hereditary category $\mathscr{B}$ of Banach spaces the collection of all improjective operators forms the largest non-identity ideal.

Proof. Let $\mu$ be the largest non-identity ideal in $\mathscr{B}$ (theorem 3.3) and let $P$ denote the collection of all improjective operators in $\mathscr{B}$. Then obviously $P \subset \mu$. Now let $T: X \rightarrow X^{\prime}$ be an arbitrary operator $\in \mu$. If possible, let $T$ be not improjective. Then there is an infinite dimensional closed subspace $Y$ of $X$ such that the restriction $\hat{T}$ of $T$ to $Y$ has a bounded inverse and $T Y$ is complemented in $X^{\prime}$. Now since $\mathscr{B}$ is hereditary, $Y \in \mathscr{B}$ and hence, by lemma $3.2, \hat{T} \in \mu$. Since $T Y$ is complemented in $X^{\prime}$, there is a continuous projection $Q$ of $X^{\prime}$ onto $T Y$. Again since $\mathscr{B}$ is hereditary, $Q \in \mathscr{B}$. We consider the map $Q \hat{T}=T^{\prime} . T^{\prime} \in \mu$ as $\hat{T} \in \mu$ and $T^{\prime}$ is obviously one-to-one and onto. Hence $I_{Y}=T^{\prime-1} T^{\prime} \in \mu$ as $T^{\prime} \in \mu$. This contradicts that $\mu$ is a non-identity ideal. Hence $T$ is improjective, i.e. $T \in P$. This completes the proof.

COROLlary 3.3. In the category $\mathscr{B}$ of all Banach spaces the ideal $P$ of improjective operators is the largest non-identity ideal.

The proof is an immediate consequence of the above theorem 3.4 as $\mathscr{B}$ is hereditary.

COROLlaRY 3.4. In the category $K$ of all reflexive Banach spaces the ideal $P$ of improjective operators is the largest non-identity ideal with $P^{*}=P$.

Proof. Since $K$ is a hereditary category, $P$ is the largest non-identity ideal in $K$ by theorem 3.4. $P^{*}=P$ follows from corollary 3.2 in view of the fact that $K$ is a regular category of reflexive Banach spaces.

COROLlaRY 3.5. In a hereditary category $Q$ of sub-projective spaces the ideal $S$ of strictly singular operators is the largest non-identity ideal.

Proof. $P$, the ideal of improjective operators of $Q$ is the largest non-identity ideal in $Q$ by theorem 3.4 as $Q$ is hereditary. Now let $T: X \rightarrow X^{\prime}$ be an arbitrary operator belonging to $P$. By theorem 1.3, $T \in S$ as $X^{\prime}$ is sub-projective. Hence $P \subset S$. This completes the proof.

COROLlary 3.6. In the category $R$ of all subprojective spaces the ideal $S$ of all strictly singular operators is the largest non-identity ideal.

PROOF. Since $R$ is a hereditary category of subprojective spaces, the proof follows from the corollary 3.5 .

COROLlary 3.7. In a hereditary category $\mathscr{H}$ of Hilbert spaces the ideal $C$ of compact operators is the largest non-identity ideal. 
PROOF. Since $\mathscr{H}$ is a hereditary category, $P$ theideal of improjective operators of $\mathscr{H}$ is the largest non-identity ideal in $\mathscr{H}$. Now by corollary $1.2, P \subset C$.

Corollary 3.8. In the category $\mathscr{H}^{\prime}$ of all Hilbert spaces the ideal $C$ of compact operators is the largest non-identity ideal.

Proof. Since $\mathscr{H}^{\prime}$ is a hereditary category of Hilbert spaces, the proof is an immediate consequence of the above corollary 3.7.

Lemma 3.3. If $I$ is an ideal in a category $\mathscr{C}$ and $I_{X} \in I$ for $X \in \mathscr{C}$, then for any object $\left.X^{\prime} \in \mathscr{C}, \operatorname{Hom}\left(X, X^{\prime}\right)\right) \subset I$ and $\operatorname{Hom}\left(X^{\prime}, X\right) \subset I$, where $\operatorname{Hom}\left(X, X^{\prime}\right)$ denotes the class of all maps from $X$ to $X^{\prime}$ in $\mathscr{C}$.

The proof is trivial.

THEOREM 3.5. In the category $U$ of all separable Hilbert spaces the ideal $C$ of compact operators is the largest ideal.

Proof. By corollary 3.7, $C$ is the largest non-identity ideal in $U$ as $U$ $U$ is a hereditary category. Let $I$ be a proper ideal in $U$ which is not contained in $C$. Then $I$ is not a non-identity ideal in $U$, i.e. there must be an infinite dimensional separable Hilbert space $X$ such that $I_{X} \in I$. Now let $X^{\prime}$ be an arbitrary infinite dimensional separable Hilbert space in $U$. Then there is an isomorphism $T$ of $X$ onto $X^{\prime}$. Now $T I_{X}=T \in I$ as $I_{X} \in I$. Hence $I_{X^{\prime}}=T T^{-1} \in I$ as $T \in I$. Thus by lemma 3.3 $\operatorname{Hom}\left(X^{\prime}, X_{1}\right) \subset I$ and $\operatorname{Hom}\left(X_{1}, X^{\prime}\right) \subset I$ for arbitrary infinite dimensional space $X^{\prime} \in U$ and arbitrary $X_{1} \in U \cdots \cdots$ (1).

Next, let $Y$ be an arbitrary finite dimensional space of dimension $n$. Now for any finite integer $k$, let $M_{k}$ be a $k$-dimensional subspace of $X$. Then the restriction $I_{X}^{\prime}$ of $I_{X}$ to $M_{k}$ is in $I$ by lemma 3.2. Let $P$ be the projection of $X$ onto $M_{k}$. Then $I_{M_{k}}=P I_{X}{ }^{\prime} \in I$ and $I_{X}{ }^{\prime} \in I$. Hence for an $n$-dimensional subspace $M_{n}$ of $X, I_{M} \in I$. Now since the dimension of $Y$ is $n$, there is an isomorphism $T^{\prime}$ of $Y$ onto $M_{n}$ and $T^{\prime}=I_{M_{n}} T^{\prime} \in I$ as $I_{M_{n}} \in I$. Hence $I_{Y}=T^{\prime-1} T^{\prime} \in I$ as $T^{\prime} \in I$. Thus by lemma 3.3, $\operatorname{Hom}\left(Y, X_{2}\right) \subset I$ and $\operatorname{Hom}\left(X_{2}, Y\right) \subset I$ for arbitrary finite dimensional space $Y \in U$ and arbitrary $X_{2} \in U \cdots \cdots(2)$.

Combining (1) and (2) we conclude $I=U$ which contradicts that $I$ is a proper ideal. This completes the proof.

LemMA 3.4. Let $X$ be an infinite dimensional Banach space and $T_{n}: X \rightarrow X$, $n=1,2,3, \cdots$ a sequence of improjective operators. Then $\left\|T_{n}-I_{X}\right\|$ cannot tend to zero as $n \rightarrow \infty$.

Proof. If possible, let $\left\|T_{n}-I_{x}\right\| \rightarrow 0$ as $n \rightarrow \infty$. Let $0<\varepsilon<1$. Then we can find a sufficiently large value $m$ of $n$ such that $\left\|T_{m}-I_{x}\right\|<\varepsilon$. Now

$$
\left\|T_{m} x\right\|=\left\|\left[I_{X}-\left(I_{X}-T_{m}\right)\right] x\right\| \geqq\left[\left\|I_{X} x\right\|-\left\|\left(I_{X}-T_{m}\right) x\right\|\right] \geqq(1-\varepsilon)\|x\|
$$


for all $x \in X$. But since $(1-\varepsilon)>0, T_{m}$ is an isomorphism of $X$ into $X$. Again since

$$
\left\|T_{m}^{*}-I_{X}^{*}\right\|=\left\|T_{m}-I_{x}\right\|<\varepsilon,
$$

we can similarly show that $T_{m}^{*}$ is an isomorphism of $X^{*}$ into $X^{*}$. Now since $T_{m}{ }^{*}$ is an isomorphism of $X^{*}$ into $X^{*}$, it is well known that $T_{m}$ is onto. Thus $T_{m}$, being an isomorphism of $X$ onto $X$, is not an improjective operator which is a contradiction. This proves the lemma.

THEOREM 3.6. If $X$ and $X^{\prime}$ are Banach spaces and $T_{n}: X \rightarrow X^{\prime}, n=1,2,3, \cdots$ is a sequence of improjective operators such that $\left\|T_{n}-T\right\| \rightarrow 0$ as $n \rightarrow \infty$ for some bounded linear operator $T: X \rightarrow X^{\prime}$, then $T$ is improjective.

ProOF. Let $\mathscr{B}$ be the category of all Banach spaces. Let $I$ be the collection of all bounded linear operators $\hat{T}$ in $\mathscr{B}$ such that for each $\hat{T} \in I$, there is a sequence $\hat{T}_{n}, n=1,2,3, \cdots$ of improjective operators such that $\left\|\hat{T}_{n}-\hat{T}\right\| \rightarrow 0$ as $n \rightarrow \infty$. Evidently $T$ of our theorem belongs to $I$. Now since $\left\|A \hat{T}_{n}-A \hat{T}\right\| \rightarrow 0$ as $n \rightarrow \infty$ and $\left\|\hat{T}_{n} B-T B\right\| \rightarrow 0$ as $n \rightarrow \infty$ whenever $\left\|\hat{T}_{n}-\hat{T}\right\| \rightarrow 0$ as $n \rightarrow \infty, A, B \in \mathscr{B}$ and $A \hat{T}_{n}$ and $\hat{T}_{n} B$ are defined, it is clear that $I$ is an ideal in $\mathscr{B}$. Because of lemma $3.4 I$ is a non-identity ideal. Hence $I \subset P$ by corollary 3.3 where $P$ is the ideal of improjective operators of $\mathscr{B}$. Hence $T \in P$. This completes the proof.

Thus all the improjective operators of a Banach space $X$ into a Banach space $X^{\prime}$ form a closed subset of the space $B\left(X, X^{\prime}\right)$ of all bounded linear operators of $X$ into $X^{\prime}$. We do not know if the improjective operators form a subspace of the space $B\left(X, X^{\prime}\right)$. However, to prove this we need only to prove that given an infinite dimensional Banach space $X, I_{X}$ cannot be expressed as the sum of two improjective operators on $X$. We do not know if the above is true.

\section{References}

[1] A. Pelczynski, 'Projections in certain Banacyh space', Studia Math. 19 (1960), 209-228.

[2] T. Kato, Perturbation theory for linear operators (Springcr-Verlag, Berlin, 1966).

[3] T. Kato, 'Perturbation theory for nullity, deficiency and other quantities of linear opeators,' J. Analyse Math. 6 (1958), 261-322.

[4] R. J. Whitley, 'Strictly singular operators and their conjugates', Trans. Amer. Math. Soc. 113 (1964), 252-261.

[5] S. Goldberg and E. Thorpe, 'On some open questions concerning strictly singular operators', Proc. Amer. Math. Soc. 14 (1963), 334-336.

[6] A. Pelczynski, 'On strictly singular and strictly co-singular operators I and II', Bull. Acad. Polon. Sci. 13 No. 1 (1965), 31-41.

[7] A. Grothendiek, 'Sur les applications linéaires faiblement compactes d'espaces du type $C(K)$ ', Canad. J. Math. 5 (1953), 129-173.

[8] S. Banach and S. Mazur, 'Zur theorie der linearen dimension', Studia Math. 4 (1933), 100-112. 
[9] M. Day, Normed linear spaces (Springer, Berlin, 1958).

[10] R. S. Philips. 'On linear transformations', Trans. Amer. Math. Soc. 48 (1940), 516-541.

[11] A. Taylor, Introduction to functional analysis (Wiley, New York, 1958).

[12] R. C. James, 'Separable conjugate spaces,' Pacific J. Math. 10 (1960), 563-571.

[13] N. Dunford and J. Schwartz, Linear operators, vol. I (Interscience, New York, 1958).

[14] A. Pelczynski and Z. Semadeni, 'Spaces of continuous functions (III)', Studia Math. 18 (1959), $211-222$.

[15] A. Sobczyk, 'Projection of the space $(m)$ on its subspace $\left(c_{0}\right)$ ', Bull. Amer. Math. Soc. 47 (1941), 938-947.

[16] S. Banach, Théorie des opérations linéaires (Warszawa, 1932).

[17] A. C. Zaanen, Linear analysis (North Holland Publicashing Co., Amsterdam, 1953).

[18] E. Lacey and R. J. Whitley, 'Conditions under which all the bounded linear operators are compact', Math. Annalen 158 (1965), 1-5.

[19] H. E. Lacey and P. D. Morris, 'Continuous linear operators on spaces of continuous functions', Proc. Amer. Math. Soc. 17 No. 4 (1966) 848-853.

[20] P. Freyd. Abelian categories, an introduction to the theory of functors (Harper and Row, New York, 1964).

[21] J. Isbell, 'Subobjects, adequacy, completeness and categories of algebras', Rozprawy Mat. 38 (1963), 1-32.

[22] G. M. Kelly, 'On the radical of a category', J. Austral. Math. Soc. 4 (1964), 299-307.,

[23] E.G. Sulgeifer, 'On the general theory of radicals in categories', Mat. Sb. 51 (93) (1960), 487500; also in Am. Math. Transl., Ser. 2, vol. 59, 150-162.

[24] E. G. Sulgeifer, 'The lattice of ideals of an object of a category I, II', Mat. Sb. 54 (96) (1961), 335-344; also in Am. Math. Transl., Ser. 2, vol. 59, 163-190.

[25] R. Schatten, Norm ideals of completely continuous operators, (Springer-Verlag, Berlin, 1960).

[26] J. W. Calkin, 'Two-sided ideals and congreunces in the ring of bounded linear operators in Hilbert space', Annals of Mathematics 42, No. 4 (1941), 839-873.

[27] I. C. Gohberg, A. S. Markus and I. A. Feldman, 'Normally solvable operators and ideals associated with them', Izv. Moldavsk. Fil. Akad. Nauk SSSR 10, No. 76 (1960), 51-69 (Russian).

School of Mathematical Sciences

Flinders University

South Australia

Present address:

Department of Mathematics

University of Queensland

St. Lucia, Brislane 4068

Australia 